\title{
An in vivo and in vitro trial of aclarubicin in metastatic breast cancer: a novel approach to the study of analogs
}

\author{
Ronald B. Natale, Robert L. Cody, Michael S. Simon, and Richard H. Wheeler* \\ Department of Internal Medicine, Division of Hematology/Oncology, University of Michigan, Ann Arbor, Michigan, USA
}

Received 4 March 1992/Accepted 27 August 1992

Summary. Aclarubicin is an anthracycline antibiotic that differs from doxorubicin in its structure, mechanism of action, and preclinical toxicity profile, especially its reduced cardiotoxicity. We therefore conducted a side-byside in vivo and in vitro trial of this agent in metastatic breast-cancer patients and their biopsied tumor specimens, respectively. Aclarubicin $\left(100 \mathrm{mg} / \mathrm{m}^{2}\right)$ was given by intravenous infusion every 3 weeks to 22 patients with objectively measurable metastatic breast cancer, 15 of whom had not previously received doxorubicin. The dose-limiting toxicity consisted primarily of leukopenia and severe nausea and vomiting. No objective response was observed in the 19 evaluable patients. After disease progression, 10 of the 15 doxorubicin-naive patients were treated with doxorubicin; 6 patients achieved a partial response, including 4 who responded to doxorubicin alone and 2 who responded to doxorubicin in combination with thiotepa and vinblastine. Tumor specimens were obtained from 14 of the 22 patients prior to the start of therapy and were tested for in vitro sensitivity to aclarubicin and doxorubicin using a soft agar colony-forming assay. Adequate colony growth occurred in 9 of 14 cultured tumor specimens. All 9 specimens, including 3 obtained from doxorubicin-naive patients, demonstrated in vitro resistance to aclarubicin. In all, 1 of 3 specimens taken from doxorubicin-naive patients demonstrated in vitro sensitivity to doxorubicin, whereas 6 tumor specimens obtained from patients who had undergone prior doxorubicin therapy demonstrated in vitro resistance. The patient whose tumor demonstrated in vitro doxorubicin sensitivity responded to a doxorubicin regimen after failing aclarubicin treatment; in vitro doxorubicin resistance correlated with clinical resistance in all cases. We conclude that aclarubicin is inactive in metastatic breast cancer at the dose and schedule used. Side-by-side

\footnotetext{
* Current address: Comprehensive Cancer Center, University of Alabama in Birmingham, University Station, Birmingham, Alabama, USA

in vivo and in vitro trials are feasible and could be useful in the development of investigational agents with activity greater than that of aclarubicin and, particularly, in the evaluation of analogs of clinically active drugs.

\section{Introduction}

Anthracycline antibiotics are among the most useful of the currently available anticancer agents [12]. Interest in aclarubicin (aclacinomycin-A; ACM-A) stems from its class similarity, structural and mechanistic differences, and diminished preclinical cardiotoxicity profile as compared with doxorubicin [2-4]. Phase I trials established $100 \mathrm{mg} / \mathrm{m}^{2}$ as a suitable dose for phase II trials using an every-3-week schedule, the side effects being dose-limiting myelosuppression and occasional mild hepatic toxicity [8]. Phase II trials in most solid tumors have been disappointing, including two studies in patients with metastatic breast cancer [5-6]. However, in the study by Forastiere et al. [5] in which no objective responses were observed, all 22 evaluable patients with breast cancer had previously received doxorubicin and may therefore have been "anthracycline-resistant." Although all patients were doxorubicin-naive in the negative study by Gockerman et al. [6], most patients were treated on a weekly schedule and the subsequent response or lack of response to doxorubicin or other agents was not reported.

Our study was designed to evaluate the activity of aclarubicin given on an every-3-week schedule to patients with metastatic breast cancer and to cross nonresponding doxorubicin-naive patients over to doxorubicin treatment so as to obtain direct data regarding cross-resistance. Furthermore, the in vitro sensitivity to aclarubicin and doxorubicin of tumor specimens obtained from patients entered into this clinical trial was evaluated using a modification of the Hamburger-Salmon human tumor colonyforming assay. 


\section{Patients and methods}

Clinical trial. A total of 22 patients with objectively measurable metastatic breast cancer gave signed informed consent and were entered in the study. Adequate marrow reserve (WBC $>4,000 / \mathrm{mm}^{3}$; granulocytes $>2,000 / \mathrm{mm}^{3}$; platelets $>150,000 / \mathrm{mm}^{3}$ ), renal function (serum creatinine $<2.0 \mathrm{mg} / 100 \mathrm{ml}$ ), hepatobiliary function (serum bilirubin, $<2.0 \mathrm{mg} / 100 \mathrm{ml}$ ) and Karnofsky performance status ( $\geq 60 \%$ ) were present in all patients. Sites of metastases among the patients varied, and most had received prior anticancer therapy (Table 1). In all, 15 patients had not previously received doxorubicin or other anthracycline therapy.

Aclarubicin was given at a dose of $100 \mathrm{mg} / \mathrm{m}^{2}$ intravenously over 30 min at 3-week intervals. Dose adjustments were made on the basis of hematologic toxicity, Doxorubicin-naive patients who continued to meet the study criteria were given doxorubicin after they had developed disease progression during aclarubicin therapy and were followed for response and assessment of cross-resistance. Single-agent doxorubicin therapy was planned, but combination drug treatment was permitted if rapid disease progression threatened the patients' survival. Patients were considered to be evaluable for response if they received two courses of therapy or if unequivocal disease progression could be documented. Standard response criteria defined in previous trials were used [7]. All patients were considered to be evaluable for toxicity if they completed a course of treatment.

In vitro trial. After informed consent had been obtained, tissues containing tumor cells were collected from all patients entered in the clinical trial with easily biopsied metastatic sites. Tumor samples were mechanically disaggregated into 2- to 3-mm fragments in Roswell Park Memorial Institute (RPMI) media containing $10 \%$ heat-inactivated fetal bovine serum (FBS), and malignant effusions or bone-marrow aspiration specimens were collected by centrifugation, fractionated over a Ficoll-Hypaque gradient, and resuspended in media. The resulting cell suspensions were filtered through a sterile wire screen to remove large fragments, washed twice in RPMI with $10 \%$ FBS, and passed through a $25-\mu \mathrm{m}$ nylon filter to remove all but single cells. Cell viability was assessed using trypan blue dye exclusion, and only viable cells were used to determine the final concentration of plated cells.

In vitro drug testing. Tumor cell suspensions were transferred to tubes prior to culture and adjusted to an appropriate final cell concentration in the presence of various concentrations of aclarubicin, doxorubicin, or media (control). Each drug was tested at three dose levels corresponding to final concentrations of $10,1.0$, and $0.1 \mu \mathrm{g} / \mathrm{ml}$. If the cell yield was too low for testing of all three drug concentrations, the highest concentrations of doxorubicin and aclarubicin were excluded. Following a 1-h exposure to media (control) or drug, cells were washed three times and seeded in agar cultures as described below.

Culture procedures. Cells were suspended in 0.3\% agar in RPMI containing $15 \%$ heat-inactivated FBS, transferrin $(10 \mu \mathrm{g} / \mathrm{ml})$, insulin $(20 \mu \mathrm{g} / \mathrm{ml})$, sodium selenite $(50 \mathrm{nM})$, L-glutamine $(2 \mathrm{mM})$, and $1 \%(\mathrm{v} / \mathrm{v})$ penicillin/streptomycin to achieve a final concentration of $5 \times 10^{5}$ cells $/ \mathrm{ml} \times 3-4 \mathrm{ml}$. A $1-\mathrm{ml}$ aliquot of this solution was pipetted over each of 3-4 petri dishes containing $1 \mathrm{mi} 0.5 \%$ agar in RPMI containing $15 \%$ FBS, L-glutamine (2 mM), and $1 \%(\mathrm{v} / \mathrm{v})$ penicillin/streptomycin. The plates were incubated in a humidified incubator in an atmosphere containing $7.5 \% \mathrm{CO}_{2}$ and were examined under an inverted microscope every other day beginning on day 9 for colony formation. Two extra "control" plates were prepared: one containing $10 \mu \mathrm{g}$ abrin/ml in the bottom agar layer was incubated [10], and one fixed with glutaraldehyde was refrigerated. The glutaraldehyde control plate had to have less than 5 "colonies" per plate for an experiment to be considered acceptable. If adequate colony growth occurred in a standard control plate (an average of $\geqslant 30$ colonies in control plates over any background colonies seen on the fixed control plates) and $<5$ colonies grew in the abrin plate, all plates were counted. Colony growth in drug-exposed plates was compared with that in control plates. Based on the pharmacokinetics of aclarubicin and previous in vitro-in vivo correlative trials $[1,9,11]$, in vitro sensitivity was defined as a concentration-dependent decrease in colony formation corresponding to $<30 \%$ colony survival at the $0.1 \mu \mathrm{g} / \mathrm{ml}$ concentration and $<5 \%$ survival at the 1.0 - or $10-\mu \mathrm{g} / \mathrm{ml}$ concentration.
Table 1. Patient's characteristics

\begin{tabular}{|c|c|}
\hline $\begin{array}{l}\text { Total number of patients on study } \\
\text { Evaluable for response } \\
\text { Evaluable for toxicity }\end{array}$ & $\begin{array}{l}22 \\
19 \\
20\end{array}$ \\
\hline Median age (range) & $59(30-77)$ years \\
\hline $\begin{array}{l}\text { Median Karnofsky performance status } \\
\text { (range) }\end{array}$ & $80(60-100)$ \\
\hline $\begin{array}{l}\text { Prior therapy: } \\
\text { Chemotherapy } \\
\text { Median number of drugs (range) } \\
\text { Doxorubicin } \\
\text { Radiotherapy } \\
\text { Hormonal therapy }\end{array}$ & $\begin{array}{l}21 \\
4(0-8) \\
7 \\
13 \\
5\end{array}$ \\
\hline $\begin{array}{l}\text { Sites of measureable diseasea: } \\
\text { Skin } \\
\text { Lung } \\
\text { Lymph node(s) } \\
\text { Liver }\end{array}$ & $\begin{array}{r}11 \\
10 \\
5 \\
5\end{array}$ \\
\hline $\begin{array}{l}\text { Toxicity: } \\
\text { Drug-related deaths } \\
\text { Hematologic: } \\
\text { WBC median (1st cycle) } \\
\text { Platelet median (1st cycle) } \\
\text { Gastrointestinal } \\
\text { Nausea/vomiting } \\
\text { (1st cycle median) }\end{array}$ & $\begin{array}{c}0 \\
2.5(0.9-5.1) \times 10^{3} / \mathrm{mm}^{3} \\
136(26-396) \times 10^{3} / \mathrm{mm}^{3}\end{array}$ \\
\hline
\end{tabular}

a 8 Patients had 2 or mores sites of measurable disease

b According to Southwest Oncology Group criteria

\section{Results}

In all, 21 of 22 patients were evaluable for toxicity and 19 were evaluable for response. The 3 unevaluable patients included 1 who died within 7 days of the start of therapy and prior to evaluation and 2 others who refused further treatment or follow-up after the first cycle. Toxicity was substantial at the dose and schedule employed for this study (Table 1). The median WBC nadir for the first cycle was $2,500 / \mathrm{mm}^{3}$, and the median nausea and vomiting score was grade 3 (Southwest Oncology Group criteria) despite the use of aggressive antiemetic regimens, including combination metoclopramide, diphenhydramine, lorazepam, and dexamethasone. The patients' acceptance of this agent was poor, with 2 patients refusing further therapy with aclarubicin after the first dose and 3 refusing all further chemotherapy for disease progression during this study because of severe (grade 4) gastrointestinal toxicity.

No objective response was observed in the 19 evaluable patients (predicted true response rate, $<15 \%, P=0.05$ ), including 12 doxorubicin-naive patients (predicted true response rate, $<22 \%, P=0.05$; see Table 2 ). In all, 10 of 12 doxorubicin-naive patients were subsequently treated with doxorubicin alone or in combination; 4 of 7 patients treated with doxorubicin alone ( $60 \mathrm{mg} / \mathrm{m}^{2}$ every 3 weeks) and 2 of 3 patients treated with doxorubicin $\left(40 \mathrm{mg} / \mathrm{m}^{2}\right)$ in combination with thiotepa $\left(12 \mathrm{mg} / \mathrm{m}^{2}\right)$ and vinblastine $\left(4 \mathrm{mg} / \mathrm{m}^{2}\right.$; TAV) every 3 weeks achieved a partial response.

Tumor specimens were obtained from 14 of 22 patients entered in the clinical trial prior to the start of therapy 


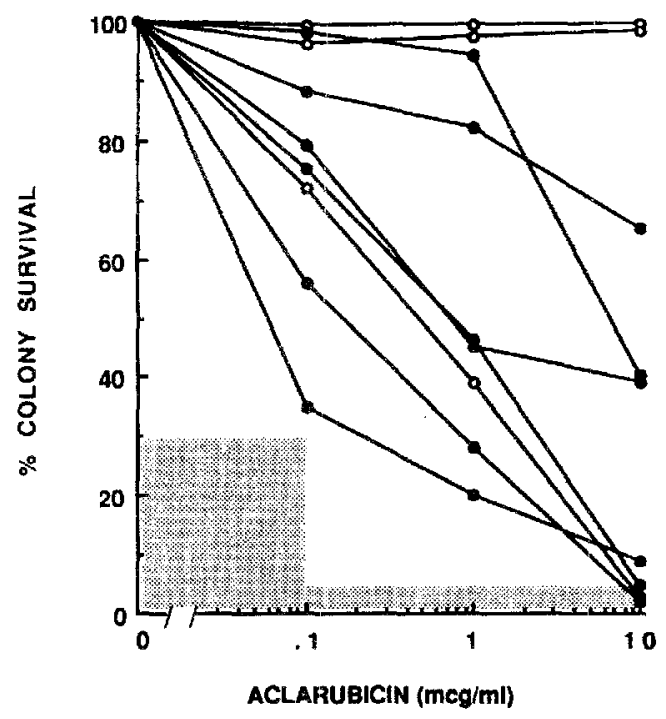

Fig. 1. Colony survival of 9 breast-cancer tumor specimens following a 1-h exposure to aclarubicin ( - , specimen from a patient who had undergone prior doxorubicin treatment; $\bigcirc$, specimen from a patient who had not previously received doxorubicin). The shaded area indicates the area into which a survival curve must fall to satisfy our definition of in vitro sensitivity

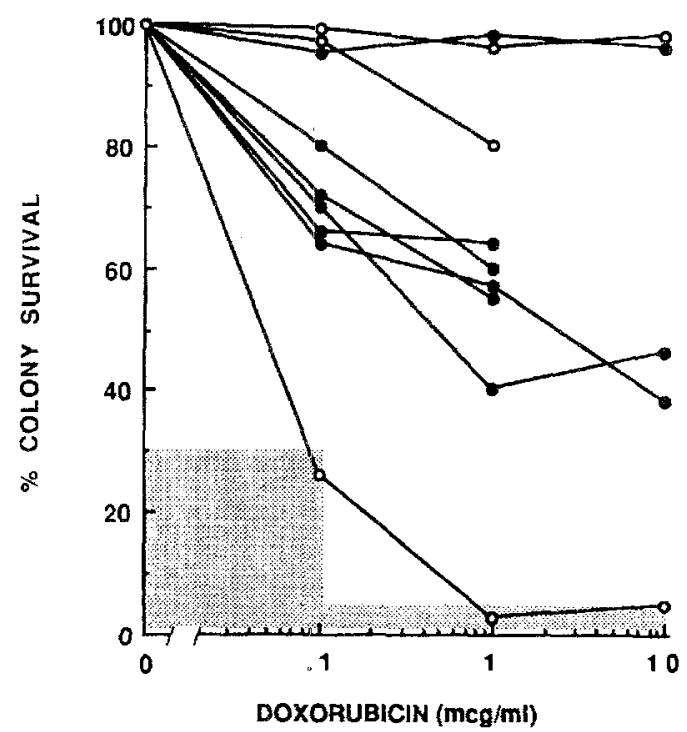

Fig. 2. Colony survival of 9 breast-cancer tumor specimens following a 1-h exposure to doxorubicin (-, specimen from a patient who had undergone prior doxorubicin treatment; $O$, specimen from a patient who had not previously received doxorubicin). The shaded area indicates the area into which a survival curve must fall to satisfy our definition of in vitro sensitivity

(6 lymph node or cutaneous metastases, 4 malignant pleural effusions, and 4 bone-marrow aspirations/biopsies of metastatic sites). Colony growth ranged from $0-147$, and "adequate" colony growth (as defined above) occurred in 9 cases (median number of control colonies, 67; range, $30-147$ ); the malignant phenotype of colonies was confirmed by examining at least 20 hematoxylin and eosinstained colonies harvested by microsyringe aspiration from a control plate in 7 of the 9 cases.
Figures 1 and 2 display the colony-survival curves generated following exposure to aclarubicin and doxorubicin, respectively. Although a concentration-dependent loss in colony survival was observed in at least 5 cases, none of the 9 tumor specimens demonstrated sufficient in vitro sensitivity to aclarubicin (as defined above) for prediction of the in vivo response. This finding correlates well with the negative clinical results of the study. In all, 6 tumor specimens obtained from patients who had developed disease progression following prior treatment with doxorubicin demonstrated in vitro resistance to doxorubicin; 1 of the 3 specimens taken from doxorubicin-naive patients demonstrated in vitro sensitivity and 2 demonstrated in vitro resistance. The patient from whom the former specimen was obtained achieved a 6 -month partial response to a doxorubicin-containing regimen (TAV), whereas the latter 2 patients failed to respond to single-agent doxorubicin.

\section{Discussion}

Aclarubicin given on an every-3-week schedule is inactive in patients with metastatic breast cancer. When we combined our findings with the results of two other trials $[5,6]$, we found no objective response among 29 patients previously treated with doxorubicin or among 22 doxorubicinnaive patients. Furthermore, in our study, 6 of 10 doxorubicin-naive patients achieved major objective responses to doxorubicin (alone or in combination) following treatment with aclarubicin. This provides strong evidence of the anthracycline sensitivity and the chemoresponsiveness of at least a portion of the patients entered in our study.

Our effort to conduct a side-by-side in vivo and in vitro trial of aclarubicin and doxorubicin using a soft agar colony-forming assay was reasonably successful in that 9 of 14 $(64 \%)$ specimens grew sufficiently well in soft agar culture for the determination of in vitro drug sensitivity. At least 5 of the 9 tumor specimens demonstrated a concentration-dependent loss in colony survival following a $1-h$ exposure to aclarubicin, confirming the cytotoxic properties of this novel anthracycline. However, none of the specimens demonstrated a loss in colony survival sufficient to satisfy our definition of in vitro sensitivity. That none of our patients showed a clinical response to aclarubicin suggests that the limits set by our working definition were reasonable. One patient whose tumor specimen demonstrated in vitro sensitivity to doxorubicin (as defined) and in vitro resistance to aclarubicin achieved a partial response to a doxorubicin-containing regimen after failing aclarubicin treatment.

In this small test sample, the results of a human tumor colony-forming assay correlated with a lack of response to chemotherapy in 11 of 11 cases and with a response in 1 of 1 case. Although these findings are not statistically meaningful, they do suggest that side-by-side in vivo and in vitro trials of new agents may be feasible and that this approach could be useful in the development of new agents and, in particular, in the evaluation of analogs of clinically active drugs. If aclarubicin had possessed greater activity, thus justifying the extension of this in vivo and in vitro study to 
an additional 15-20 doxorubicin-naive patients and their tumor specimens, important data regarding cross-resistance could have been obtained. We recommend further testing of this approach for the development and clinical testing of new analogs.

Acknowledgements. This work was supported in part by contract NO1CM-07405 and grant RO1-CA-38741 from the Division of Cancer Treatment, National Cancer Institute.

\section{References}

1. Alberts DS, Salmon SE, Chen HSG, Moon TE, Young L, Surwit EA (1981) Pharmacologic studies of anticancer drugs with the human tumor stem cell assay. Cancer Chemother Pharmacol 6: 253

2. Clinical brochure (July 1979) Aclacinomycin A (NSC-208734). Investigational Drug Branch, Cancer Therapy Evaluation Program, Division of Cancer Treatment, National Cancer Institute, Bethesda, Maryland

3. Danchev D, Slioussartchouk V, Paintrand M, Hayat M, Bourut C, Mathe G (1979) Electron microscopic studies of the heart and light microscopic studies of the skin after treatment of golden hamsters with Adriamycin, detouribicin, AD 32, and aclacinomycin. Cancer Treat Rep 63:931
4. Dorr RT, Bozak KA, Shipp NG, Hendrix M, Alberts DS, Ahman F (1988) In vitro rat myocyte cardiotoxicity model for antitumor antibiotics using adenosine triphosphate/protein ratios. Cancer Res 48: 5222

5. Forastiere AA, Budman DR, Richards F, Aisner J, Weinberg V, Wood WC (1985) Phase II trial of aclacinomycin in advanced breast cancer: a Cancer and Leukemia Group B study. Cancer Treat Rep 67: 1137

6. Gockerman JP, Raney M, Logan T (1985) Phase II evaluation of aclacinomycin in advanced breast cancer: a Southeastern Cancer Study Group trial. Cancer Treat Rep 69: 1029

7. Hayward JL, Carbone PP, Heuson JC, Kamaoka S, Segaloff A, Rubens RD (1977) Assessment of response to therapy in advanced breast cancer. Cancer 39:1289

8. Ogawa M, Inagki J, Horikoski, Inoue E, Chinen T, Ueoka H, Nagura E (1979) Clinical study of aclacinomycin-A. Cancer Treat Rep 63: 931

9. Salmon SE, Liu RM, Casazza AM (1981) Evaluation of new anthracycline analogs with the human tumor stem cell assay. Cancer Chemother Pharmacol 6: 103

10. Salmon SE, Liu R, Hayes C, Persaud J, Roberts R (1983) Usefulness of abrin as a positive control for the human tumor clonogenic assay. Invest New Drugs 1: $277-281$

11. VonHoff DD, Casper J, Bradley E, Sandbach J, Jones D, Makuch R (1981) Association between human tumor colony-forming assay results and response of an individual patient's tumor to chemotherapy. Am J Med 70: 1027

12. Wadler S, Fuks JZ, Wiemik PH (1986) Phase I and II agents in cancer therapy: 1. Anthracyclines and related compounds. J Clin Pharmacol 26: 491 